\title{
Images of the month: Lower-back pain in a young man with HLA B27 - not always spondyloarthritis!
}

\author{
Authors: Ashok Kumar, ${ }^{A}$ Ashish Sharma ${ }^{B}$ and Anunay Agarwal ${ }^{\mathrm{C}}$
}

Fig 1. X-ray of pelvis and magnetic resonance imaging of sacroiliac joint showing lumbosacral transitional vertebra. a) X-ray of pelvis: anteroposterior projection showing elongated right transverse process of fifth lumbar vertebra (bordered by asterisks); pseudoarticulation between the right transverse process of $L 5$ vertebra and ala of sacrum (arrows); non-fusion of posterior elements of $L 5$ vertebra (encircled area) suggestive of spina bifida occulta. b) and c) Magnetic resonance imaging of sacroiliac joint: pseudoarticulation between the right transverse process of $L 5$ vertebra and ala of sacrum (arrows).
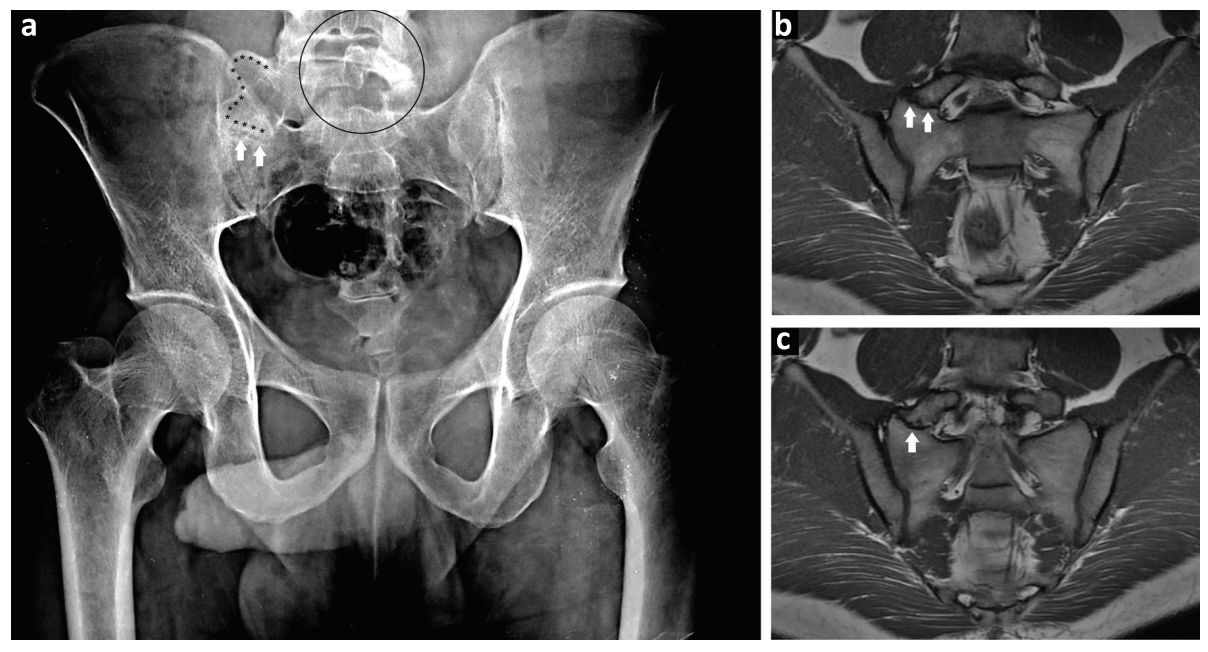

Presence of human leucocyte antigen (HLA) B27 in a patient with lower-back pain should not be considered synonymous with axial spondyloarthritis. Other causes of back pain should be ruled-out by careful evaluation. Lumbosacral transitional vertebra is a common congenital malformation of spine which frequently leads to mechanical back pain. We present a young man with lower-back pain who had HLA B27. He was labelled with axial spondyloarthritis elsewhere and was given etanercept without benefit. Further evaluation revealed that he had lumbosacral transitional vertebra and spina bifida occulta. No evidence of sacroiliitis was found. Etanercept was stopped and he was started on physiotherapy protocol for transitional vertebra, with which he improved remarkably. This case highlights the need for greater awareness among clinicians about lumbosacral transitional vertebra, a finding which is frequently missed. Presence of HLA B27 can be coincidental, as in our case.

KEYWORDS: Back pain, HLA B27, transitional vertebra, spina bifida occulta, spondyloarthritis

DOI: $10.7861 /$ clinmed.2020-0070

Authors: ${ }^{\text {A }}$ consultant, Fortis Hospital, Noida, India; ${ }^{B}$ consultant and department head, Fortis Flt Lt Rajan Dhall Hospital, New Delhi, India; Consultant, Aakash Hospital, New Delhi, India

\section{Case presentation}

A 28-year-old man presented with lower-back pain, progressing over the previous 6 months. Forward bending and weight lifting aggravated the pain; rest provided relief. Human leucocyte antigen (HLA) B27 was positive and he was prescribed etanercept, subcutaneous injections of $50 \mathrm{mg}$ weekly, elsewhere. He presented to our hospital after taking 10 weekly injections of etanercept with no benefit. Tenderness was observed in the right sacroiliac joint area. Investigations showed erythrocyte sedimentation rate of $12 \mathrm{~mm}$ in the first hour (normal range $<30$ ) and C-reactive protein of $2.8 \mathrm{mg}$ per litre (normal range $<10$ ). X-ray of the pelvis revealed an elongated right transverse process of fifth lumbar vertebra and pseudoarthrosis with right ala of sacrum (Fig 1a). There was non-fusion of posterior elements of $L 5$ vertebra, suggestive of spina bifida (Fig 1a). Magnetic resonance imaging (MRI) of pelvis confirmed the presence of pseudoarthrosis (Figs $1 \mathrm{~b}$ and c); there was no sacroiliitis. The patient was diagnosed with lumbosacral transitional vertebra with spina bifida occulta. Etanercept was stopped and physiotherapy protocol for transitional vertebra was initiated. At the 4-month follow-up, the patient's back pain improved remarkably.

\section{Discussion}

Lumbosacral transitional vertebra is a common congenital malformation of spine. Sacralisation of fifth lumbar vertebra or lumbarisation of first sacral vertebra is frequently observed. This 
results in pseudoarticulation between the ala of sacrum and the transverse process of the vertebra above. It leads to back pain due to intervertebral disc degeneration, facet joint arthropathy and mechanical stress at the level of pseudoarticulation. ${ }^{2}$ The pain may mimic that of inflammatory sacroiliitis due to axial spondyloarthritis. Our patient had HLA B27 in addition to lowerback pain, consequently, he was diagnosed with spondyloarthritis elsewhere. However, he did not have the inflammatory type of back pain and acute phase reactants and sacroiliac joints on MRI were normal. These points were against the diagnosis of spondyloarthritis. Non-steroidal anti-inflammatory drugs and physiotherapy are the mainstay of treatment. ${ }^{3}$

This case highlights the need for greater awareness among clinicians about lumbosacral transitional vertebra, a finding which is frequently missed. Presence of HLA B27 can be coincidental, as in our patient.

\section{References}

1 Jancuska JM, Spivak JM, Bendo JA. A review of symptomatic lumbosacral transitional vertebrae: Bertolotti's syndrome. Int ] Spine Surg 2015;9:42.

2 Jain A, Agarwal A, Jain S, Shamshery C. Bertolotti syndrome: a diagnostic and management dilemma for pain physicians. Korean J Pain 2013;26:368-73.

3 Brenner AK. Use of lumbosacral region manipulation and therapeutic exercises for a patient with a lumbosacral transitional vertebra and low back pain. J Orthop Sports Phys Ther 2005;35:368-76.

Address for correspondence: Dr Ashish Sharma, Department of Rheumatology, Fortis Hospital, Sector 62, Noida, Uttar Pradesh 201301, India.

Email: ash.blueney@gmail.com 